\title{
A New Authentication Method for the Valuables Logistics Personnel
}

\author{
Linan Fan, Qin Xue, Wei Wu
}

Department of Information Engineering, Shenyang University, Shenyang, 110044, China

\author{
Keywords: Palm vein, Authentication, Valuables, Logistics, Block, Partial Least Squares.
}

\begin{abstract}
Palm vein recognition is a new biometric identification technology, mainly used for authentication. This paper for the first time applies it to supervise and manage clients, couriers, the warehouse administrators in the valuables logistics. Aiming at when valuables are lost, damaged, it guarantees responsibilities to individuals. we use contactless palm vein recognition technology based on the block and partial least squares, and do experiments on CASIA gallery. The experimental results shows when the block size is $4 \times 4$ and principal component number is 240 , the recognition performance is best, then the correct recognition rate is $99.34 \%$, the deterrent rate is $0.66 \%$, the false reject rate is $0.60 \%$, the recognition time is $0.0379 \mathrm{~s}$. Obviously, this algorithm has practical value and meets the application requirements of the valuables logistics.
\end{abstract}

\section{Introduction}

With the development of social economy, more and more valuables such as jewelry, antiques, calligraphy and painting, furniture, luxury watches, high-grade health care products, high value stamps, electronic digital products, high-tech instruments use courier as the transport mean. The service market demand of the valuables logistics is rising sharply.

Also, this is the frequent accidents "high risk" area where express was loss and damage. Most consumers never take the initiative to send courier insured, they generally agreed that if the goods are damaged, courier companies should follow the actual damages. When the valuables in the logistics process are lost, damaged, both prone to disputes. Because the sender is not insured, the example of courier companies refuse to fully compensate is very common. In addition, some items such as documents is significant, once lost, it will give consumers a lot of trouble and inconvenience.

Obviously, the valuables missing in logistics caused so serious dissatisfaction among consumers that consumers lose confidence in the logistics enterprises[1]. Valuables logistics service mode has become a key social issue that must be addressed. Traditional authentication methods such as ID, text messaging, phone numbers, passwords, job number, user name are easily lost, forgotten, copied and at theft risks, so these methods has been unable to meet the needs of valuables logistics.

To overcome these drawbacks, this paper uses biometric identification technology[2].Biological characteristics include face, iris, palmprint, fingerprints, palm vein, finger vein, voice, hand shape and so on. Palm vein identification as an emerging biometric technology[3], has unique advantages: with respect to the palmprint recognition, palm vein is less susceptible to peeling of the palms[4]; relative to the finger vein or fingerprint, palm vein has a larger area[5]; with respect to the human face, palm vein recognition can easily distinguish twins[6]; relative to the iris, palm veins collection methods make it easier for users to accept[7], etc.

Therefore, we uses palm vein recognition technology to supervise and manage clients, couriers, the warehouse administrators in the valuables logistics. When valuables are lost, damaged, it guarantees responsibilities to individuals.

\section{Authentication process}

\section{Valuables logistics methods}

This paper designed a new type of valuables supervision and management scheme. We combine the palm vein recognition technology with SPS mobile phone bar gun currently used[8], apply palm vein recognition technology to supervise operate valuables logistics personnel, such as: customers, 
couriers, warehouse manager, etc., manage valuables itself using SPS phone bar gun. The program specific operation is as follows:

In the receiving process, after inspection and packaging, courier use their own palm vein feature to login SPS mobile gun, scan the customer's palm vein characteristics to determine the sender's identity, then scan the express barcode and upload the data.

In express warehousing process, the warehouse administrator check goods information in front of receiving courier, login SPS mobile gun using own palm vein features, scan the express barcode and upload the data to prove the express warehousing. In the warehouse, operators are required the palm vein identity authentication before maintain, loading and unloading, handling valuables. When discharging, the warehouse administrator use palm vein features to land SPS mobile phone bar gun, scan the express barcode and upload the data to prove the express have been out of the storehouse.

In the sending process, courier use their own palm vein feature to login SPS mobile gun, scan the customer's palm vein characteristics to determine the recipient's identity, remind customers face to face acceptance, after the inspection is completed, ask the customer autographed recipients to sign the waybill column, then scan the express barcode and upload the data.

This scheme complete synchronously the management of the personnel and express, improve the staff vigilance, ensure the valuables logistics safety, reduce the incidence of the accident about items lost, damaged, impersonator.

\section{Palm vein recognition process}

Palm vein recognition is divided into two stages: registration and certification process, as shown in Fig.1.

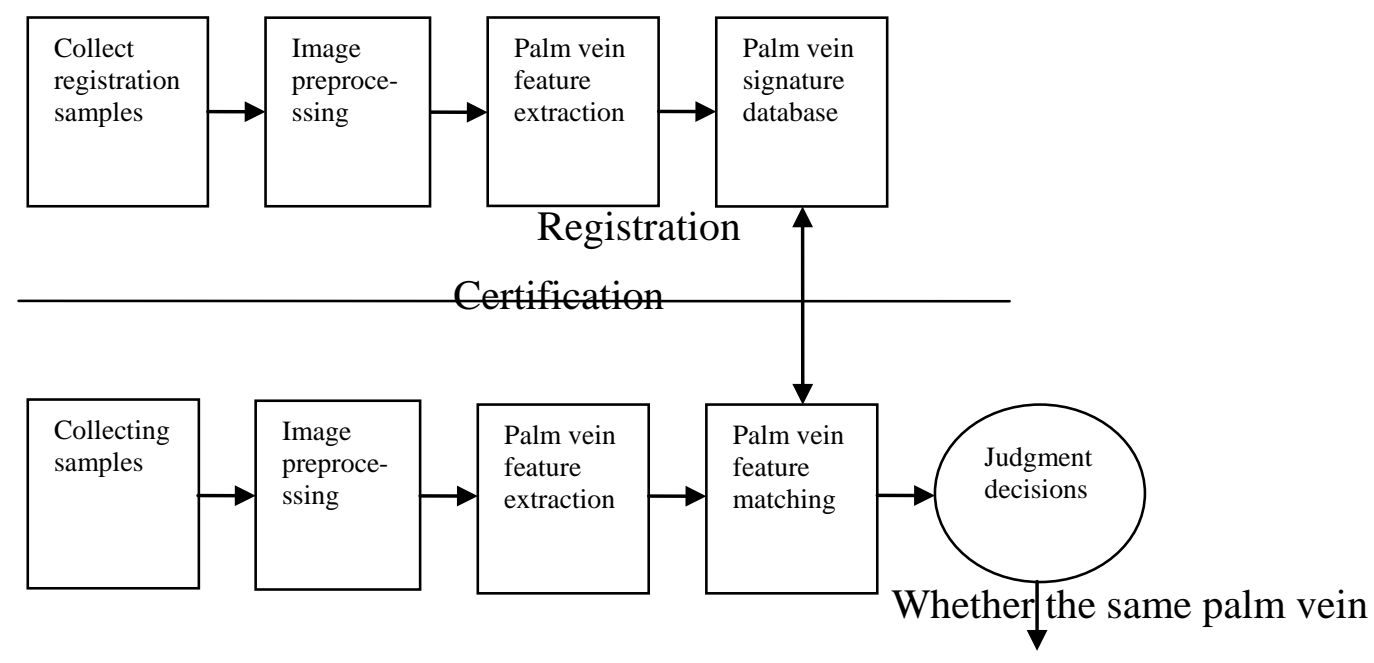

Fig.1: Palm vein recognition process

\section{Palm vein recognition algorithm}

\section{Image acquisition}

In the absence of voluntary, we collect palm vein image of clients, couriers, the warehouse administrators and promise to protect their privacy. Do experiments on Chinese academy of sciences institute of automation multi-spectral palmprint Image database V1.0(CASIA). The palmprint gallery can display palm vein, is a generic palm veins gallery. Choose 100 people in the gallery, 50 people left hand, 50 people right hand, 6 pieces each, a total of 600 .

\section{Image preprocessing}

The article adopts the method of literature[9] to locate ROI, as shown in figure 2.The positioning method is to find a stable feature points on the palm: the valley point of the index finger and middle finger, the valley point of ring finger and little finger. To a certain extent, it adjust image rotation, translation and scaling problem caused by the non-contact shooting. 

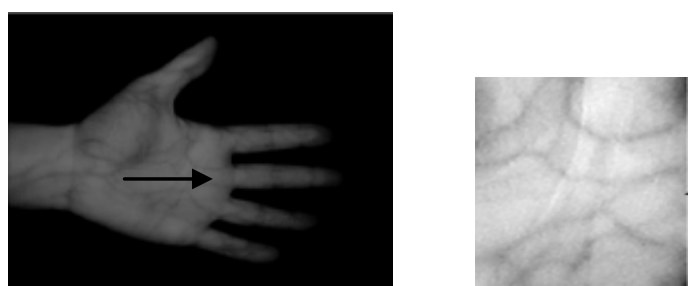

Fig.2: Schematic diagram of ROI location determination

\section{Feature extraction.}

The article adopts the method of literature [10] to feature extraction, first with a block algorithm for fast image dimensionality reduction, then use partial least squares algorithms to extract classification subspace, in which image gray value variation is large, and category information is most relevant.

Non-overlapping sub-block means the palm vein image $A, \mathrm{a} \times n$ matrix, is divided into $\mathrm{p} \times q$ sub- blocks of the image matrix.

$$
A=\left[\begin{array}{cccc}
A_{11} & A_{12} & \cdots & A_{1 q} \\
A_{21} & A_{22} & \cdots & A_{2 q} \\
\vdots & \vdots & \vdots & \ddots \\
A_{p 1} & A_{p 2} & \cdots & A_{p q}
\end{array}\right]
$$

Wherein: each of the sub-image $A_{i j}(i=1,2, \cdots, p ; j=1,2, \cdots, q)$ is $\mathrm{m}_{1} \times n_{1}, \quad \mathrm{~m}=p \times m_{1}, n=q \times n_{1}$. The sub-image gray value is replaced by the mean gray value of the image block.

$\mathrm{u}_{r h}=\frac{1}{m_{1} n_{1}} \sum_{r=1}^{m_{1}} \sum_{h=1}^{n_{1}} h(x, y) \quad(r=1,2, \cdots, p ; h=1,2, \cdots, q)$

Wherein: ${ }^{u_{r h}}$ represents any sub image, $\mathrm{h}(x, y)$ represents the gray value of point $(x, y)$.All sub-image average consists low-dimensional image matrix of $\quad \mathrm{p} \times q$ dimensional:

$$
I=\left[\begin{array}{cccc}
u_{11} & u_{12} & \cdots & u_{1 q} \\
u_{21} & u_{22} & \cdots & u_{2 q} \\
\vdots & \vdots & \vdots & \ddots \\
u_{\mathrm{p} 1} & u_{p 2} & \cdots & u_{p q}
\end{array}\right]
$$

With the block image participating in PLS operation.

Assuming that the independent variable matrix $X$ consists of column vector of the image, $X=\left(x_{1}, x_{2}, \cdots, x_{p}\right)^{T}$.Where $x_{t}(t=1,2, \cdots, p)$ is the column vector consisting of the $t^{t}$ image.Put $\mathrm{P}_{\text {images }}$ into $G$ classes, Provided the dependent variable matrix $Y=\left(\mathrm{y}_{1}, y_{2} \cdots, \mathrm{y}_{p}\right)^{T}, \mathrm{y}_{t}=\left(y_{t 1}, y_{t 2}, \cdots, y_{t G}\right)^{T}(t=1,2, \cdots, p)$. When $\mathrm{y}_{t}$ belongs to the class $i(i=1,2, \cdots, G), \quad \mathrm{y}_{t j}=\left\{\begin{array}{ll}1 & j=i \\ 0 & j \neq i\end{array} \mathrm{j}=1,2, \cdots, G\right.$. It is assumed that $X$ and $Y$ are standardized variables, their mean are 0 and variance are 1 .

Step1: extract the first pair ingredients from two groups of variables: $T_{1}, U_{1}$. $T_{1}=w_{11} X_{1}+w_{12} X_{2}+\cdots+w_{1 p} X_{p}=w_{1}^{T} X$

$$
U_{1}=v_{11} Y_{1}+v_{12} Y_{2}+\cdots+v_{1 q} Y_{q}=v_{1}^{T} Y
$$

(5) $T_{1}$ and $U_{1}$ should be as large as possible to carry the variation information, and the $T_{1}$ and $U_{1}$ reached the maximum correlation degree.That is,they must meet the following conditions: $\operatorname{MAX}\left(\operatorname{cov}\left(T_{1}, U_{1}\right)\right)=\sqrt{\operatorname{var}\left(T_{1}\right) \operatorname{var}\left(U_{1}\right)} \mathrm{r}\left(T_{1}, U_{1}\right)$

(6) Wherein: $\operatorname{cov}(\cdot)$ is the covariance operator, $\operatorname{var}(\cdot)$ is the variance operator, $r(\cdot)$ operator is the correlation coefficient. Constraints : $\left\|v_{1}\right\|^{2}=1\left\|w_{1}\right\|^{2}=1$ 
Step2: Calculate the score vector of the first pair components: ${ }_{1}, \mathrm{u}_{1}, \mathrm{t}_{1}=X w_{1} ; u_{1}=Y v_{1}$. The covariance of first pair component $T_{1}$ and $U_{1}$ can be calculated by the inner product of first pair component sub-vector $\mathrm{t}_{1}$ and $\mathrm{u}_{1}$.According to Lagrange multiplier method ,formula (4) corresponding to the optimal solution $w_{1}$ is the matrix $w_{1}^{T} X^{T} Y v_{1}$ standard feature vector of largest

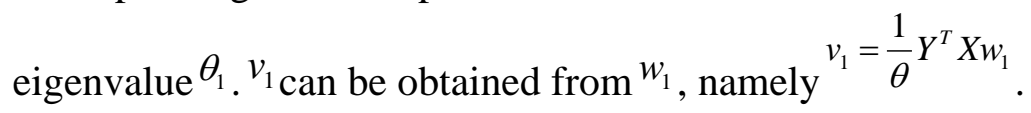

Step3: ${ }^{w_{1}}$ is called the first spindle, ${ }_{1}{ }_{1}$ is called the first PLS component principal. Do $X$ and $Y$ linear regression on $\mathrm{t}_{1}$ : $X=t_{1} P_{1}^{\prime}+X_{1}$

(7) $Y=t_{1} R_{1}^{\prime}+Y_{1}$

Regression coefficient: $P_{1}=\frac{X^{T} t_{1}}{\left\|t_{1}\right\|^{2}} \quad R_{1}=\frac{Y^{T} t_{1}}{\left\|t_{1}\right\|^{2}}$

$X_{1}$ and $Y_{1}$ are the residual matrices. If the equation meets the preset accuracy, the algorithm stops. Otherwise, $X_{1}$ and $Y_{1}$ instead of $X$ and $Y$ extract the second PLS components. If, in accordance with requirements of the precision, a PLS main components: $t_{1}, \cdots, t_{m}$, then:

$X=t_{1} P_{1}^{\prime}+t_{2} P_{2}^{\prime}+\cdots+t_{m} P_{m}^{\prime}+X_{m}$

$Y=a_{1} t_{1}+a_{2} t_{2}+\cdots+a_{m} t_{m}+Y_{m}$

(10) Step4: model about $Y$ and $X_{1}, X_{2}, \cdots, X_{p}$ can be written as:

$Y=\beta_{1} X_{1}+\beta_{2} X_{2}+\cdots+\beta_{m} X_{m}+Y_{m}$

(11) This is the interpretation of $X$ on $Y$, also, it is the hand vein recognition feature extraction model. The feature vectors $\beta_{1}, \beta_{2}, \cdots \beta_{m}$ are a set of coordinate coefficients. The ROI image projects to these $m$ feature vectors, which can obtain a set of coordinates. It represents the position of the image in the subspace, also is the basis of classification.

\section{Feature matching}

After feature extraction, image ${ }^{p}$ obtained a set of position coordinates ${ }^{F_{p}}$ in the subspace, which is used as the feature vector matching. Matching distance is to calculate the Euclidean distance between image feature vector $F_{p}$ of $p$ and image feature vector $F_{q}$ of $q$,denoted by: ${ }^{D i s_{p q}}=\left|F_{p}-F_{q}\right|$.According to distribution curve of within-class matching and category matching,we calculate the threshold $\boldsymbol{t}$.If feature vectors Euclidean distance of two image meet: ${ }^{D i s}\langle t$, palm veins are considered to belong to the same person, otherwise, the person is rejected.

\section{Experimental results and analysis}

In order to verify the effectiveness of the method, we use the CASIA gallery for experiments. The first three pieces per person are for training, last three for testing.100 Class palm vein samples match 90,000 times, in which the within-class match 900 times, between-class match 89,100 times. Calculation the recognition performance of the algorithm, respectively, when block size is $4 \times 4$ and the number of principal components varies from 40 to 240 (step 20). The results are shown in Table 1. 
Table1: FAR, FRR, CRR, Time for different principle component numbers on CASIA database

\begin{tabular}{|c|c|c|c|c|c|c|}
\hline \multicolumn{7}{|c|}{ principle component numbers } \\
\hline & 40 & 60 & 80 & 100 & 120 & 140 \\
\hline $\begin{array}{l}\text { FA } \\
\text { R }\end{array}$ & 11.68 & 16.95 & 15.32 & 14.58 & 11.48 & 11.48 \\
\hline $\begin{array}{l}\text { FR } \\
\text { R }\end{array}$ & 20.73 & 13.20 & 12.93 & 11.13 & 10.93 & 5.73 \\
\hline $\begin{array}{l}\mathrm{CR} \\
\mathrm{R}\end{array}$ & 82.25 & 83.08 & 84.70 & 85.45 & 88.53 & 88.07 \\
\hline $\begin{array}{l}\text { Tim } \\
\mathrm{e}\end{array}$ & 0.0034 & 0.0056 & 0.0080 & 0.0103 & 0.0131 & 0.0184 \\
\hline \multicolumn{7}{|c|}{ principle component numbers } \\
\hline & 160 & 180 & 200 & 220 & 240 & \\
\hline $\begin{array}{l}\text { FA } \\
\text { R }\end{array}$ & 7.43 & 4.26 & 3.15 & 1.41 & 0.66 & \\
\hline $\begin{array}{l}\text { FR } \\
\mathrm{R}\end{array}$ & 5.00 & 3.73 & 1.67 & 1.33 & 0.60 & \\
\hline $\begin{array}{l}\mathrm{CR} \\
\mathrm{R}\end{array}$ & 92.59 & 95.75 & 96.86 & 98.59 & 99.34 & \\
\hline $\begin{array}{l}\text { Tim } \\
\mathrm{e}\end{array}$ & 0.0189 & 0.0224 & 0.0279 & 0.0285 & 0.0379 & \\
\hline
\end{tabular}

Analysis: the recognition performance is best when principal component number is 240, then the FAR is $0.66 \%$, FRR is $0.60 \%$, CRR is $99.34 \%$, the recognition time is 0.0379 s. This algorithm has practical value and meets the application requirements of the valuables logistics.

\section{Conclusions}

In this paper, palm vein recognition technology obtain a higher recognition rate, low false rejection rate, meet the real-time application requirements. It can be applied to supervise and manage clients, couriers, the warehouse administrators in the valuables logistics. When valuables are lost, damaged, it guarantees responsibilities to individuals. The introduction of palm vein recognition technology also makes the staff vigilance, to a certain extent, reduce the incidence of the accident about items lost, damaged, impersonator. Therefore, this initiative is not only of great theoretical significance, but also has broad application prospects.

\section{Acknowledgements}

This research work was supported by Education department of Liaoning province (L2013444).

\section{References}

[1] Yongqiang Kong, Yu Han. Delay time,damage to the good, S.F complained by many consumers[J]. Goods and Quality.2010,(1):3.

[2] Jian A K, Ross A, prabhakar S.An introduction to biometric recognition. IEEE Transactions on Circuit and System for Video Technology.2004,14(1): 4-20.

[3] The world's leading biometrics - palm vein identification perspective. http://www.21csp.com.cn/html/View_2009/07/05/JSWZ1894.shtml

[4] Kar-Ann Toh, How-Lung Eng, Yuen-Siong Choo et al. Identity verification through palm vein and crease texture. Lecture notes in Computer science, 2006: 546-553.

[5] Weixin Bian,Deqin Xu. Complex algorithm of fingerprint image thinning[J]. Chinese journal of image and graphics,2011,16(6) : 1015-1021. 
[6] Shimin Wang, Jihua Ye, Tao Deng, etc. Two-dimensional tree complex wavelet uncertainty weighted fusion of face recognition[J].Chinese journal of image and graphics,2012,17(8) : 995-1001.

[7] Yong Chen, Jingqian Huang, Jianan Zheng.Our private courier express optimization of operational processes[J]. Logistics Engineering and Management,2013,35(3):121-127.

[8] Wang X H, Dong Q K. An kind of iris recognition algorithm based on 2D-PLDA and wavelet subband[J].Journal of Image and Graphics,2011,16 (1):59-65.

[9] Nan Wang. Palm vein feature extraction and recognition research[D]. Shenyang: Shenyang University of Technology,2011.

[10] Wei Wu. Palm vein recognition technology research[D]. Shenyang: Shenyang University of Technology,2013. 